\title{
Intratunical injection of stromal vascular fraction prevents fibrosis in a rat model of Peyronie's disease
}

\section{Fabio Castiglione ${ }^{* \dagger+}$ (D), Petter Hedlund ${ }^{\top * *}$, Emmanuel Weyne*, Lukman Hakim* ${ }^{* \dagger}$, Francesco Montorsi ${ }^{\ddagger}$, Andrea Salonia ${ }^{\ddagger}$, Trinity J. Bivalacqua ${ }^{\S}$, Dirk De Ridder*, Uros Milenkovic* ${ }^{*}$, David Ralph ${ }^{\dagger}$, Giulio Garaffa ${ }^{\dagger}$, Asif Muneer ${ }^{\dagger+}$, Steven Joniau* and Maarten Albersen* On behalf of the Trauma and Reconstructive Urology Working Party of the European Association of Urology (EAU) Young Academic Urologists (YAU)}

*Laboratory for Experimental Urology, Organ Systems, Department of Development and Regeneration, University of Leuven, Leuven, Belgium, 'Institute of Urology, University College of London Hospital, London, UK, ${ }^{t}$ Division of Oncology/Unit of Urology, Urological Research Institute, IRCCS Ospedale San Raffaele, Milan, Italy, \&James Buchanan Brady Urological Institute, Department of Urology, Johns Hopkins Medical Institutions, Baltimore, MD, USA, "Department of Clinical and Experimental Pharmacology, Lund University, Lund, **Division of Drug Research, Department of Medical and Health Sciences, Linköping University, Linköping, Sweden, "tDepartment of Urology, Airlangga University / Dr Soetomo General Hospital, Surabaya, Indonesia, and "thivision of Surgery and Interventional Science, NIHR Biomedical Research Centre, University College London Hospital, London, UK

\section{Objective}

To investigate whether local injection of autologous adipose stromal vascular fraction (SVF) can prevent the development of fibrosis and elastosis in the tunica albuginea (TA) using a rat model of the acute phase of Peyronie's disease $(\mathrm{PD})$.

\section{Methods}

A total of 24 male 12-week-old Sprague-Dawley rats were divided into three equal groups: sham; PD without treatment (transforming growth factor- $\beta$ [TGF $-\beta]$ ); and PD treated with SVF 1 day after disease induction. Sham rats received two injections of vehicle into the TA 1 day apart. TGF $-\beta$ rats received TGF- $\beta 1$ injection and injection of vehicle 1 day later. SVF rats received TGF- $\beta 1$ injection, followed by SVF 1 day later. One month after treatment, all rats underwent measurement of intracavernosal pressure and mean arterial pressure during electrostimulation of the cavernous nerve. The rats were then killed and penises were harvested for histology and Western blot analysis.

\section{Results}

Erectile function was moderately reduced in the TGF- $\beta$ group and was significantly improved after SVF treatment $(P<0.05)$. $\mathrm{PD}$ rats developed areas of fibrosis with a significant upregulation of collagen III, collagen I and elastin protein expression. These fibrotic changes were prevented when treated with SVF.

\section{Conclusions}

Local injection of SVF may represent treatment for the acute phase of PD.

\section{Keywords}

sexual dysfunction, penile plaque, adipose stem cell, \#Peyronies, \#erectiledysfunction

\section{Introduction}

Peyronie's disease (PD) is an acquired benign fibrotic disorder involving the tunica albuginea (TA) of the corpora cavernosa of the penis [1]. The disease is characterized by the formation of a fibrous plaque in the TA containing disarranged depositions of collagen and elastin, which form during a painful phase of inflammation: the acute phase [2].
An accepted hypothesis regarding the pathophysiology of PD is that, during the acute phase, impaired clearance of the inflammation can lead to abnormal wound healing, evolving into a gradually permanent fibrotic reaction: the chronic phase [2]. This phase is characterized by scar tissue retraction, calcification and occasionally ossification. Eventually, this condition can result in a permanent and painless penile deformity [3], which may present as a 
curvature, waist deformity, or a complex deformity, with a combination of wasting, rotation and curvature, resulting in impaired or impossible intercourse. It is frequently accompanied by severe and difficult-to-treat erectile dysfunction [4]. Currently, there are no evidence-based treatment options to halt the disease in the acute phase [5].

Recently, several studies have suggested a possible role of mesenchymal stem cells (MSCs) in the treatment of corpus cavernosum fibrosis [6] and PD [7-10]. Although the exact mechanisms behind the anti-fibrotic properties of MSC therapy remain to be elucidated, the leading theory is that MSCs act as a 'drug-store', influencing various profibrotic characteristics simultaneously. Most preclinical studies suggest that MSCs work through immunomodulation, thereby limiting the host response to injury and preventing the onset of fibrosis $[11,12]$.

In a previous study [7], our group showed the efficacy of human adipose tissue-derived MSCs (ADSCs) in preventing fibrosis in a rat model of acute-phase PD. ADSCs are isolated as part of the aqueous fraction derived from enzymatic digestion of lipoaspirate (the product of liposuction). This aqueous fraction, a combination of ADSCs, endothelial precursor cells, endothelial cells, macrophages, smooth muscle cells, lymphocytes, pericytes and pre-adipocytes, among others, is known as the stromal vascular fraction (SVF) [1318]. Recent advances in the area of tissue regeneration have put SVF on a par and at times even above ADSCs [19]. For instance, in a study of erectile function in a rat model of cavernous nerve $(\mathrm{CN})$ injury, SVF treatment showed superior statistically significant results compared with ADSC treatment alone, especially in smooth muscle/collagen ratio and in endothelial cell content [20]. Similar results were reported in an animal study comparing ADSCs and SVF as treatment for multiple sclerosis [21].

In the present study, we investigated the effects of early local injection of SVF on plaque formation and erectile function in a validated rat model for the acute phase of PD.

\section{Methods}

\section{Ethical Approval}

All experiments on animals and human tissues were approved by the ethics committee of Katholieke Universiteit Leuven (Leuven, Belgium) (P 272/2014). The sample size of 24 was calculated, considering three groups, a statistical power of 0.9 , an effect size of 2, and an $\alpha$ level 0.05 (G*Power 3.1).

\section{Animals}

Male Sprague-Dawley rats $(n=27 ; 12$ weeks old; $300-350$ g; Charles River Laboratories, Wilmington, MA, USA) were used. Rats were housed in pairs in 12-h reversed-cycle lighting with ad libitum access to food and water. I.p. ketamine $(75 \mathrm{mg} / \mathrm{kg})$ and xylazine $(50 \mathrm{mg} / \mathrm{kg})$ were used for anaesthesia for the surgical procedures [22]. Amoxicillin (50 mg/kg i.p.) was administered $1 \mathrm{~h}$ prior to the surgical procedures as prophylaxis. The rats were killed using carbon dioxide asphyxia as described in local regulations.

\section{Study Design}

The rats were randomly divided into three equal groups. The sham group $(n=8)$ underwent injection of $50-\mu \mathrm{L}$ vehicle (citrate buffer) in the dorsomedial aspect of the right midshaft TA with a Hamilton microlitre syringe after opening the buck fascia as previously described [7]. The remaining 16 rats were injected with recombinant $0.5 \mu \mathrm{g}$ of TGF- $\beta 1$ in 50 $\mu \mathrm{L}$ vehicle [7]. After 1 day, all rats underwent laparotomy incision and resection of the para-testicular adipose tissue. The same day, the rats received a second injection with either PBS (200 $\mu \mathrm{L}$; sham and TGF- $\beta$ groups) or autologous SVF in PBS (1 million cells in $200 \mu \mathrm{L}$; SVF group).

Four weeks after treatment, all rats underwent in vivo erectile function evaluation, after which the rats were killed and the penises harvested for histological analysis and for protein extraction.

\section{Isolation of Stromal Vascular Fraction}

The rats underwent laparotomy incision and resection of the para-testicular adipose tissue. The adipose tissue was rinsed with PBS, minced into small pieces, then incubated in a solution containing $0.075 \%$ collagenase type IA (Sigma-Aldrich, St Louis, MO, USA) for $1 \mathrm{~h}$ at $37^{\circ} \mathrm{C}$, with a vigorous shake for $15 \mathrm{~s}$ at 20 -min intervals. The top lipid layer was removed, and the remaining liquid portion was centrifuged at $1000 \mathrm{~g}$ for $10 \mathrm{~min}$ at room temperature. The pellet was treated with $160 \mathrm{mM} \mathrm{NH} 4 \mathrm{Cl}$ for $10 \mathrm{~min}$ to lyse red blood cells. The cells were then washed, centrifuged, and the pellet was resuspended in PBS. The nucleated cells from the pellet were counted and diluted to 5000 cells per $\mu \mathrm{L}$ in saline, of which $200 \mu \mathrm{L}$ were kept on ice until injection [23].

\section{Erectile Function Measurement}

Intracavernosal pressure (ICP) response to electrostimulation of the $\mathrm{CN}$ was used to evaluate erectile function [7]. Briefly, under anaesthesia, the right $\mathrm{CN}$ was exposed and the right crus of the corpus cavernosum was identified and cannulated with a heparinized $(200 \mathrm{U} / \mathrm{mL}) 25-\mathrm{G}$ needle connected to a pressure transducer. The $\mathrm{CN}$ was activated $(2.5,5$ and $7.5 \mathrm{~V})$ by platinum electrodes connected to a stimulator at $20 \mathrm{~Hz}$ for $50 \mathrm{~s}$ [7]. The nerve was stimulated once per voltage, and a resting period of 2 min was allowed for nerve recovery between stimulations. Mean arterial pressure (MAP) was recorded by carotid artery cannulation [7]. 


\section{Histological Analysis of Tissue}

The penile midshaft at the level of the injection site was harvested, fixed, and further processed for histology.

Haematoxylin and eosin and Masson's trichrome staining procedures were performed according to a standard protocol, previously described $[7,22,24,25]$.

\section{Western Blot Analysis}

Western blot analysis was performed as previously described $[7,22,24,25]$ for the detection of collagen I, collagen III and elastin proteins at the level of the penile midshafts. Glyceraldehyde-3-phosphate dehydrogenase (GADPH) was used as an internal standard. Primary antibodies were $\mathrm{Rb}$ anti-collagen III (1:1 000; Abcam Inc., Cambridge, MA, USA), Ms anti-elastin (1:500, Abcam) and Rb controls against GADPH (1:1 000, Santa Cruz Biotechnology, Santa Cruz, CA, USA), Rb anti-collagen I (1:500; Abcam Inc.) $[7,22,24,25]$.

\section{Statistical Analysis}

The results were analysed using PRISM v.4 (GraphPad Software, San Diego, CA, USA) and expressed as mean and standard deviation of the mean. Multiple groups were compared using one-way ANOVA, followed by the StudentNewman-Keuls test for post hoc comparisons. Statistical significance was set at $P<0.05[25,26]$.

\section{Results}

No rat developed infection or died after the surgical procedures.

Rats in the TGF- $\beta$ group displayed a deposition of amorphic matrix and a haphazard organization of collagen fibres in the TA, which extended into the subtunical corpus cavernosum (Fig. 1). These morphological results were corroborated by quantitative Western blot analysis, which revealed an increased protein content of collagen III/collagen I compared with the sham group $(P<0.05$ for both; Fig. 2$)$. In the SVF

Fig. 1 Histology. Representative photomicrographs of Masson's trichrome and haematoxylin and eosin (H \& E) staining in midshaft sections of rat

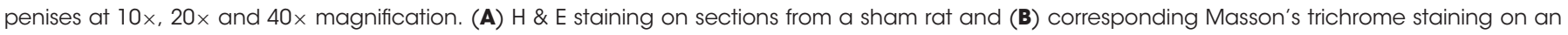

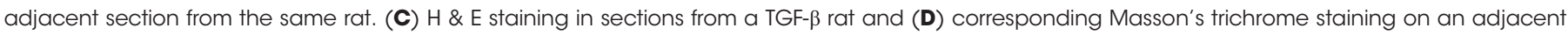
section from the same rat. (E) H \& E sections from a stromal vascular fraction (SVF) rat and (F) corresponding Masson's trichrome staining on an

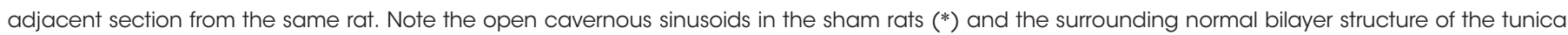

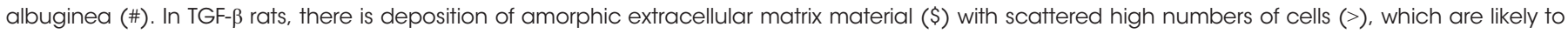
be fibroblasts based on their spindle-shaped morphology and relationship with the extracellular matrix. In the SVF rat, there is an increase in extracellular matrix deposition (+); however, collagen fibres seem better organized and the sinusoid structure is largely preserved. GADPH, glyceraldehyde 3-phosphate dehydrogenase.

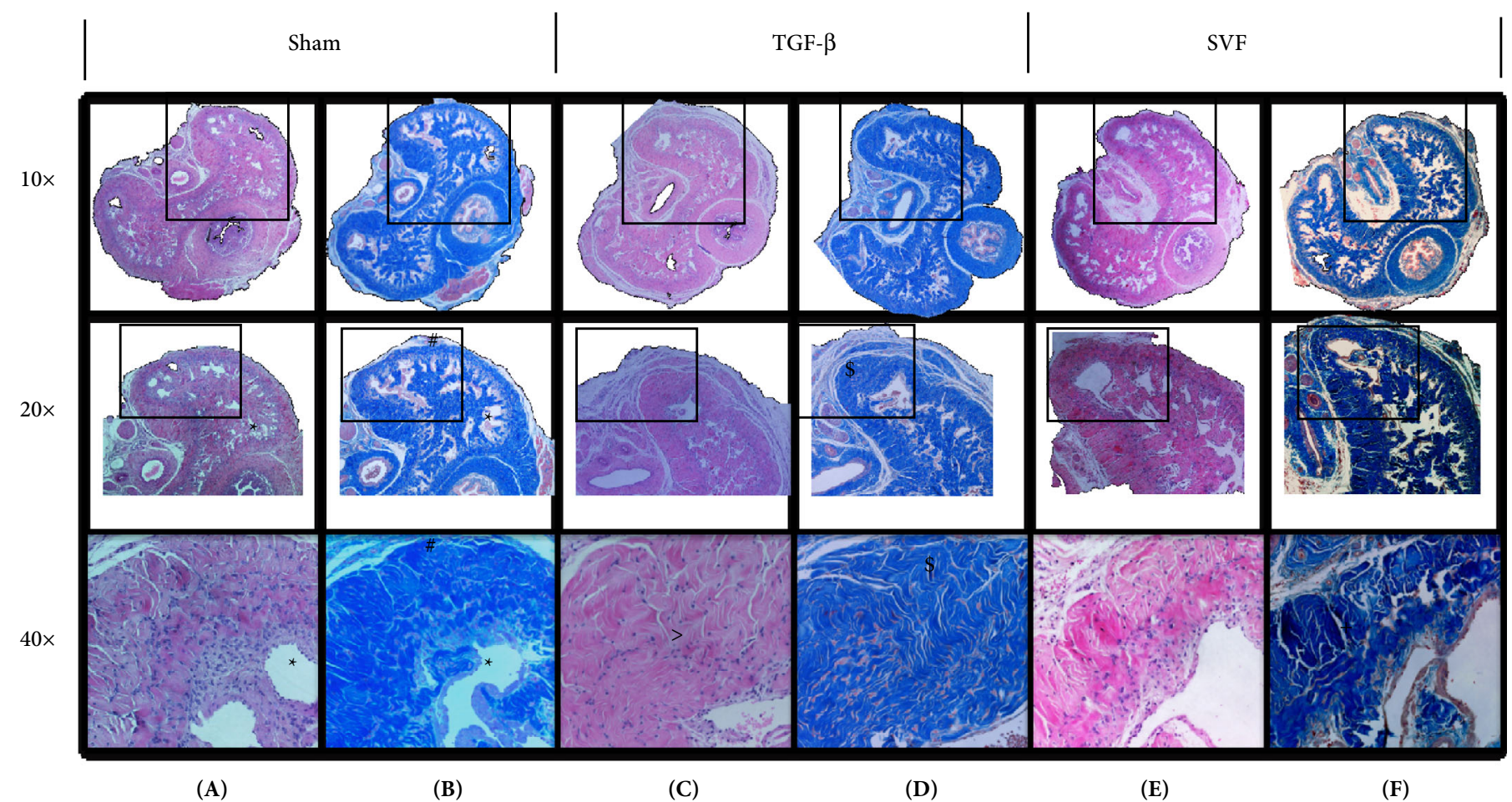


Fig. 2 Western blot analysis for collagen III, collagen I and elastin. (A) Representative chemiluminescence images of blotted membranes containing protein extracts of all three groups. Double bands are attributable to binding of antibodies to glycosylated and non- glycosylated forms of these molecules. (B) Summarized protein expression levels for elastin \# $P<0.05$ vs sham group in ANOva with post hoc Student-Newman-Keuls analysis. (C) Summarized protein expression levels for collagen I $+P<0.05$ vs both stromal vascular fraction ( SVF)-treated and sham rats in ANOVA with post hoc Student-Newman-Keuls analysis. (D) Summarized protein expression levels for collagen III $+P<0.05$ vs both sham and SVF-treated rats in ANovA with post hoc Student-Newman-Keuls analysis. (E) Collagen III and I expression ratio * $P<0.05$ vs both sham and TGF- $\beta$ in ANOVA with post hoc StudentNewman-Keuls analysis.

(A)

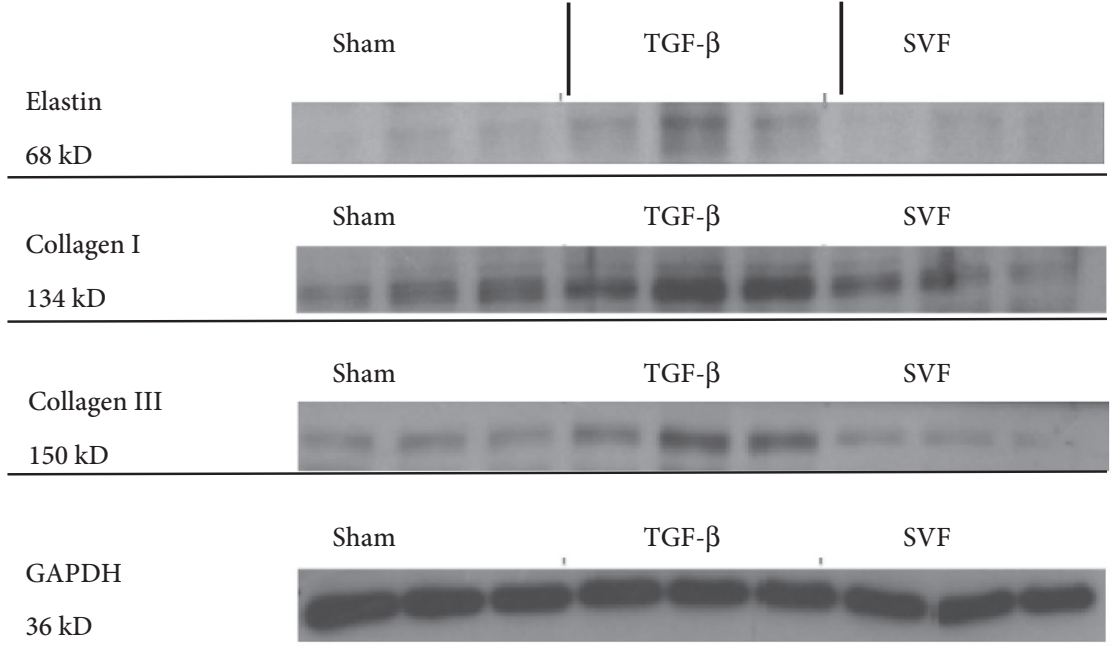
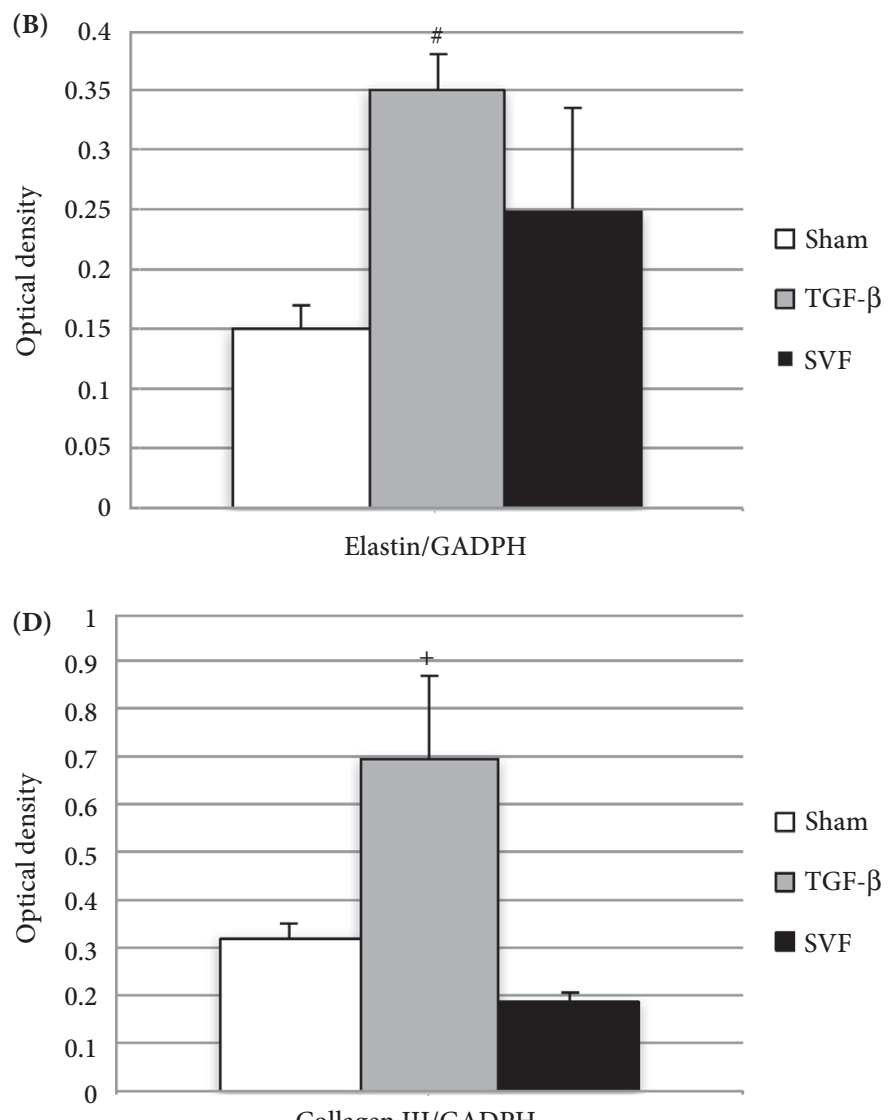
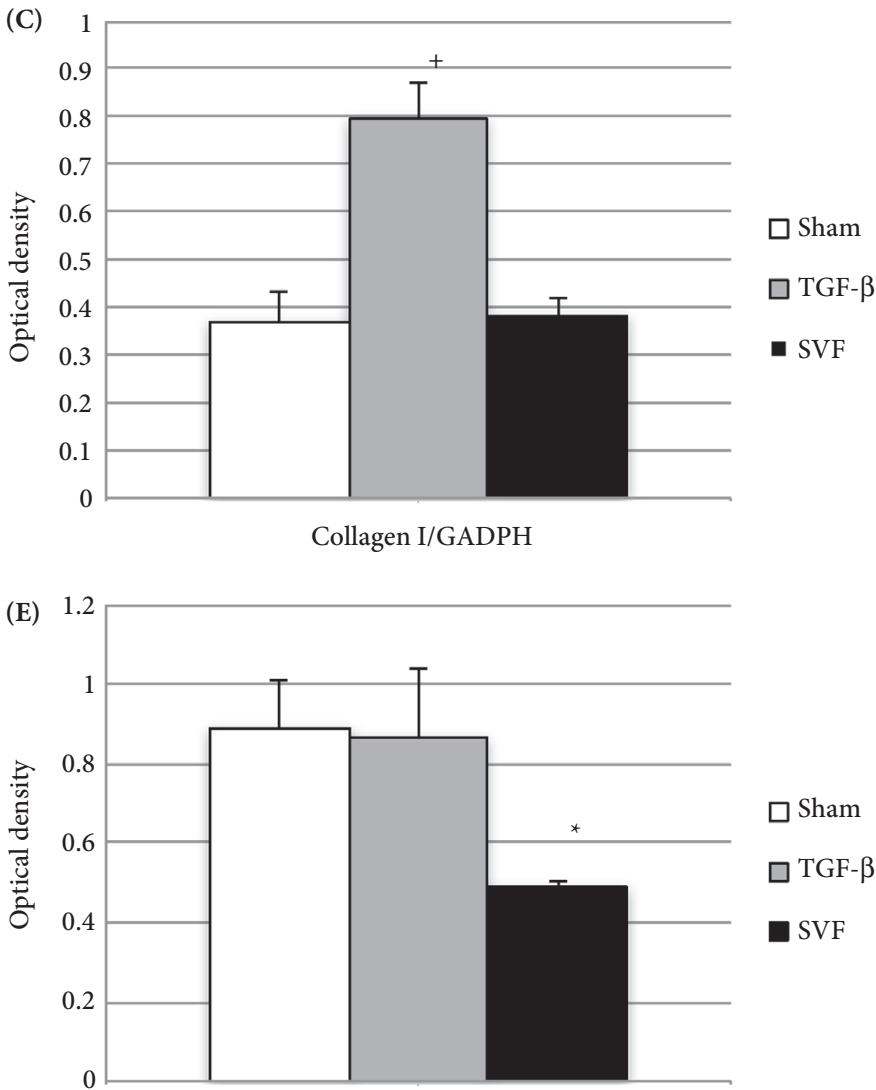

Collagen II/Collagen I 
group, the overall structure of the TA and collagen I and III expression of the penile shafts was similar to that of sham rats (Fig. 3). Penile shafts from TGF- $\beta$ rats showed more elastin expression than those from the sham group $(P<0.05)$ but no difference compared with the SVF group $(P<0.05$; Fig. 3). Interestingly, the collagen III/collagen I ratio was lower in the SVF group than in the sham and TGF- $\beta$ groups $(P<0.05)$. Quantitatively, the protein content of collagen III was reduced by $72.8 \%$ after SVF injection, as shown by Western blot analysis.

The morphological findings were supported by the functional studies. A significant difference was noted in ICP and ICP/ MAP ratio in response to $\mathrm{CN}$ electrostimulation among the three groups at $2.5,5$ and $7.5 \mathrm{~V}(P<0.05$ for all voltage $)$ 4 weeks after vehicle or SVF injection (Figs 3 and 4). Rats injected only with TGF- $\beta 1$ showed a moderate but statistically significant diminished response to $\mathrm{CN}$ electrostimulation compared with sham rats at all voltages. Treatment with SVF improved erectile function, as evidenced

Fig. 3 Erectile function measurement: summarized data comparing erectile function measurements in sham TGF- $\beta$ rats and rats treated with stromal vascular fraction (SVF) at various voltages during cavernous nerve electrostimulation. (A) Intracavernosal pressure (ICP). (B) ICP normalized over mean arterial pressure (MAP). +: $P<0.05$ vs both sham and stromal vascular fraction (SVF) rats in ANOVA with post hoc StudentNewman-Keuls analysis.
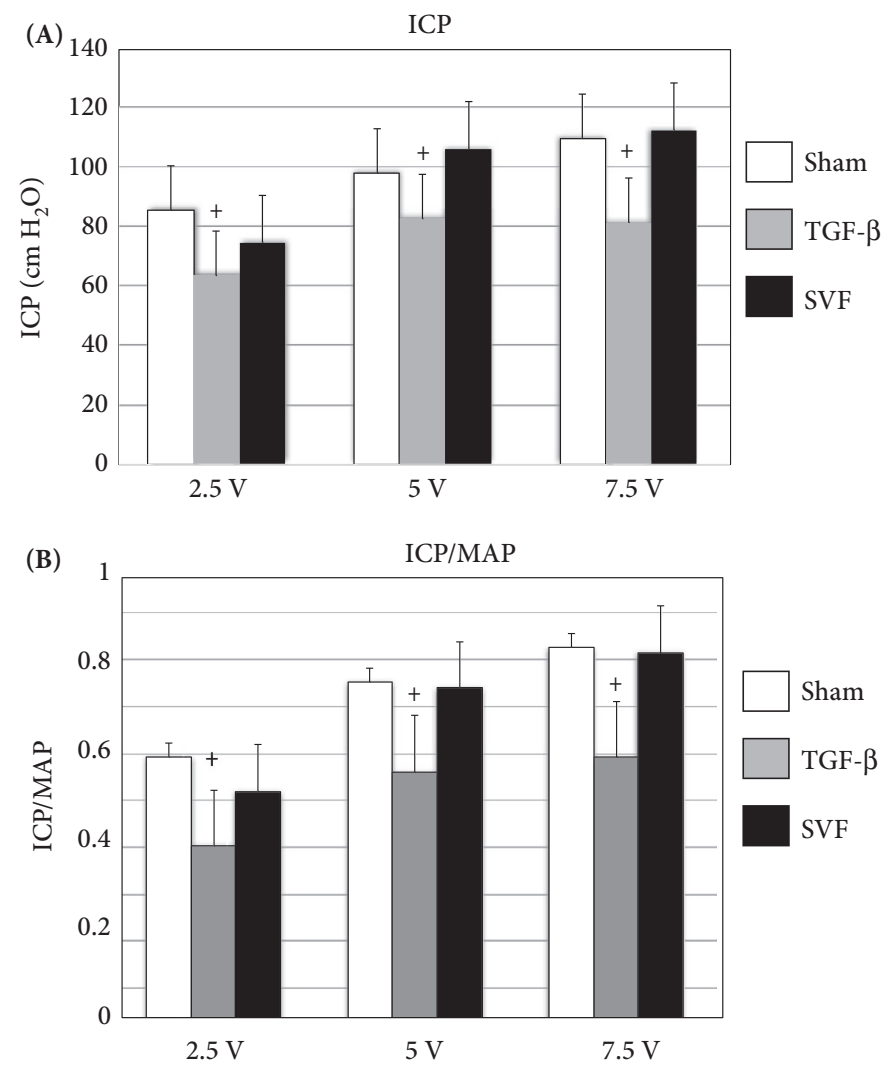

by increased ICP/MAP compared with TGF- $\beta$ at all voltages $(P<0.05)$. Quantitatively, the SVF treatment improved the ICP/MAP compared with TGF- $\beta$ treatment by $130 \%$ at $2.5 \mathrm{~V}$ $(P=0.02)$, by $132 \%$ at $5 \mathrm{~V}(P<0.001)$ and by $137 \%$ at $7.5 \mathrm{~V}$ $(P<0.001)$. No differences were noted between sham and SVF rats at all voltages $(P>0.05)$.

\section{Discussion}

Our results provide evidence that SVF reduces TA fibrosis in a rat model that is generally accepted to mimic the acute phase of PD. SVF is a heterogeneous fraction containing mature endothelial cells, vascular smooth muscle cells, macrophages, regulatory $\mathrm{T}$ cells, fibroblasts and a large population of stem/progenitor cells [13-18]. Several preclinical and clinical studies have explored the efficacy of adipose SVF in several types of fibrotic disease, and also in animal models of erectile dysfunction and incontinence, showing positive results and a similar efficacy to ADSC treatment [13-18]. There are multiple advantages of using adipose SVF rather than ADSCs in the clinical setting. Firstly, unlike ADSCs, adipose SVF is easily acquired and re-injected within a short time frame, without the need for any cell separation or culturing conditions, reducing the risk of genetic instability and teratogenesis [27]. Secondly, SVF has minimal contact with reagents, making it comparatively safe and subject to the fulfilment of fewer regulatory criteria [27]. In efforts to improve the yield of SVF isolation in the clinical setting, many companies have developed automatic processing systems, which are able to improve the isolation process by reducing the human element contamination, while still adhering to the current Good Manufacturing Practices. Many of these companies are actively pursuing clinical trials to validate their devices and technologies clinically, while also providing cellular therapy to patients in need, such as the upcoming STAR trial (Celution ${ }^{\circledR}$ - Cytori Therapeutics, ClinicalTrials.gov Identifier: NCT02396238) for the treatment of scleroderma, which has recently received an investigational device exemption from the US Food and Drug Administration and is currently ongoing [28].

To date, three preclinical studies have evaluated ADSCs injected locally as a therapy for PD [7-9]. The in vivo efficacy of ADSCs was tested using the most common model for PD: the model based on the local injection of TGF- $\beta 1$ into the TA of rats. In our previous study [7], we showed that in the acute, or inflammatory phase of the disease, xenograft injection of human ADSCs into the affected area prevents the formation of fibrosis and elastosis in the tunica and corpus cavernosum and restores erectile function. In this previous study, we injected the human ADSCs 1 day after the TGF- $\beta 1$ treatment, aiming to mimic the early phase of the disease, which is characterized by inflammation, penile pain, curvature progression and no stable identifiable fibrotic plaque [7]. After 1 month, rats injected with TGF- $\beta 1$ 
Fig. 4 Representative example traces of mean arterial pressure ( MAP) and intracavernosal pressure ( ICP) recordings during $7.5 \mathrm{~V}$ cavernous nerve electrostimulation: (A) sham rat; (B) TGF- $\beta$; (C) stromal vascular fraction (SVF).
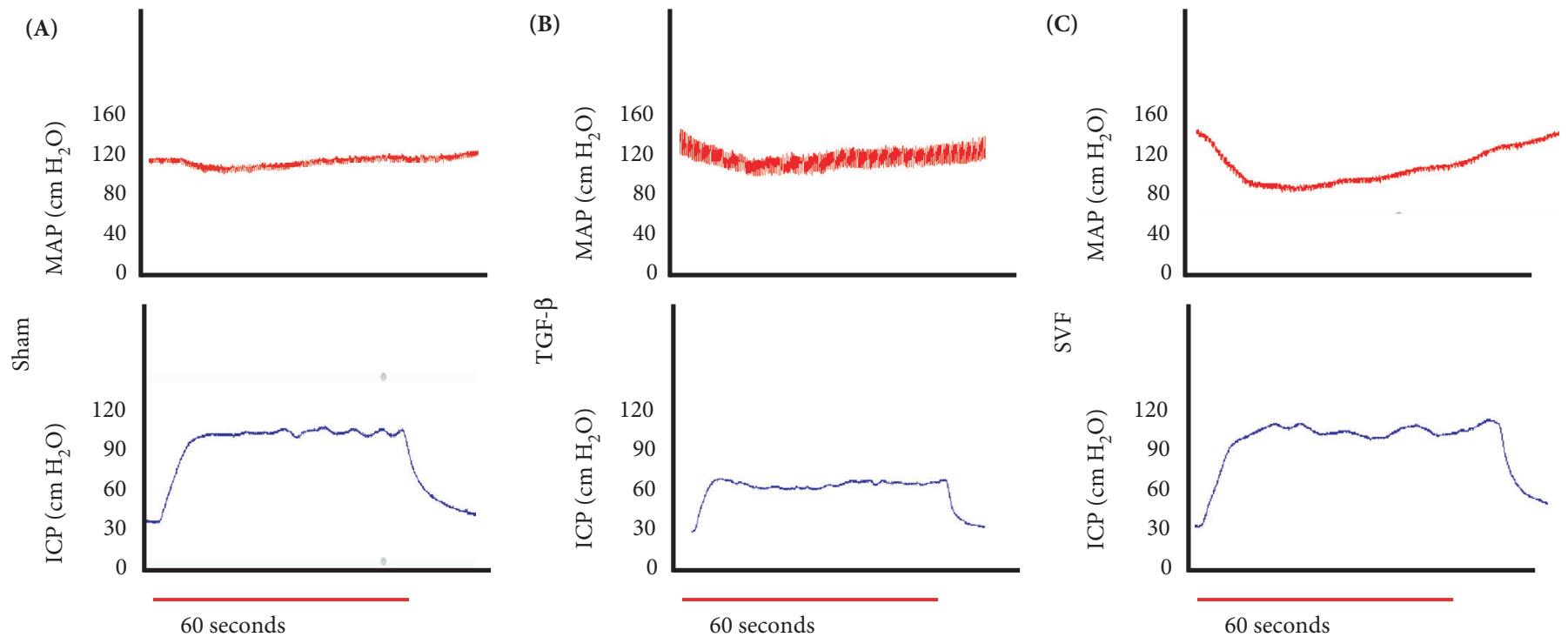

displayed extensive TA and corporal fibrosis and elastosis at the injection site, together with impaired erectile function [7]. Our results were in line with the two preclinical studies performed by Gokce et al. [8,9]. These three studies showed consistently that TA injection of ADSCs resulted in significantly lower TA fibrosis and in a better erectile function response compared with the rats treated only with TGF- $\beta 1[8,9]$.

In the present study, we aimed to assess the efficacy of SVF in the acute phase of PD using the same animal model and the same study design used in our previous experiment. Similarly to our previous results [7], the rats injected with TGF- $\beta$ developed TA fibrosis, characterized by a diffuse and disorganized deposition of elastin and both collagen III and I. These changes resulted functionally in a severe impairment of erectile function upon $\mathrm{CN}$ electrostimulation. Based on our results, SVF is able to reduce the collagen III and collagen I content in the TA of the treated rats and to ameliorate their erectile function with similar efficacy to ADSC treatment.

The present study has some inherent limitations including the lack of characterization of the injected cell types. However, others studies, using a similar isolation protocol, have examined the exact composition of SVF [23]. In addition, this study was conducted with a limited time frame for functional evaluation and we were therefore unable to assess whether the changes induced by cellular therapy persisted over a longer time. More importantly, we studied administration of SVF only in the inflammatory phase of the disease. It is known that only a small portion of patients with PD present in the early phases of the disease and therefore this study should be regarded as a proof of principle that SVF therapy is able to halt the natural development of PD plaques. Whether this cellular therapy is able to reduce established PD plaques remains to be elucidated. Further experimentation is now being conducted, targeting the effects of SVF in the chronic disease state in this model. Despite these limitations, we were able to show functional and histological improvement of SVF in PD rats, providing a translationally interesting new avenue in autologous cellular therapy for PD.

In conclusion, this study is the first to test SVF in an animal model of PD. In the acute phase of the PD, injection of SVF into the affected area prevents the formation of fibrosis in the TA. The present data, together with the availability of routine autograft SVF isolation procedure therapy, represent an encouraging translational potential of this treatment strategy.

\section{Acknowledgements}

We thank Arianna Bettiga, Fabio Benigni, Giorgia Colciago, Rita Van Bree, Catherien Luyten, Goedlieve Verbist and Petra Steven for their technical contributions to the experiments in this study.

\section{Conflict of Interest}

This study was funded by the European Society for Sexual Medicine grant for basic medical research 2011 awarded to Fabio Castiglione and Maarten Albersen and by the European Urological Scholarship Programme awarded to Fabio Castiglione. Asif Muneer is supported by the National Institute for Health Research, Biomedical Research Centre at University College London Hospital. All authors declare no competing financial interests. 


\section{References}

1 Garaffa G, Trost LW, Serefoglu EC, Ralph D, Hellstrom WJ. Understanding the course of Peyronie's disease. Int J Clin Pract 2013; 67: 781-8

2 Ostrowski KA, Gannon JR, Walsh TJ. A review of the epidemiology and treatment of Peyronie's disease. Res Rep Urol 2016; 8: 61-70

3 Sherer BA, Levine LA. Contemporary review of treatment options for Peyronie's disease. Urology 2016; 95: 16-24

4 Yafi FA, Hatzichristodoulou G, DeLay KJ, Hellstrom WJ. Review of management options for patients with atypical Peyronie's disease. Sex Med Rev 2017; 5: 211-21

5 Joice GA, Burnett AL. Nonsurgical interventions for Peyronie's Disease: update as of 2016. World J Mens Health 2016; 34: 65-72

6 Soebadi MA, Moris L, Castiglione F, Weyne E, Albersen M. Advances in stem cell research for the treatment of male sexual dysfunctions. Curr Opin Urol 2016; 26: 129-39

7 Castiglione F, Hedlund P, Van der Aa F et al. Intratunical injection of human adipose tissue-derived stem cells prevents fibrosis and is associated with improved erectile function in a rat model of Peyronie's disease. Eur Urol 2013; 63: 551-60

8 Gokce A, Abd Elmageed ZY, Lasker GF et al. Intratunical injection of genetically modified adipose tissue-derived stem cells with human interferon alpha- $2 \mathrm{~b}$ for treatment of erectile dysfunction in a rat model of tunica albugineal fibrosis. J Sex Med 2015; 12: 1533-44

9 Gokce A, Abd Elmageed ZY, Lasker GF et al. Adipose tissue-derived stem cell therapy for prevention and treatment of erectile dysfunction in a rat model of Peyronie's disease. Andrology 2014; 2: 244-51

10 Hellstrom WJ, DeLay KJ, Abdel-Mageed AB. Can adipose-derived stem cells be used in the treatment of urethral stricture disease? Eur Urol 2016; 70: $1042-3$

11 Caplan AI, Correa D. The MSC: an injury drugstore. Cell Stem Cell 2011; 9: $11-5$

12 Meirelles Lda S, Fontes AM, Covas DT, Caplan AI. Mechanisms involved in the therapeutic properties of mesenchymal stem cells. Cytokine Growth Factor Rev 2009; 20: 419-27

13 Atalay S, Coruh A, Deniz K. Stromal vascular fraction improves deep partial thickness burn wound healing. Burns 2014; 40: 1375-83

14 Ataman MG, Uysal CA, Ertas NM, Bayraktar N, Terzi A, Borman H. The effect of adipose stromal vascular fraction on transverse rectus abdominis musculocutaneous flap: an experimental study. J Plast Surg Hand Surg 2016; 50: 272-80

15 Bourin P, Bunnell BA, Casteilla L et al. Stromal cells from the adipose tissue-derived stromal vascular fraction and culture expanded adipose tissue-derived stromal/stem cells: a joint statement of the International Federation for Adipose Therapeutics and Science (IFATS) and the International Society for Cellular Therapy (ISCT). Cytotherapy 2013; 15: 641-8

16 Doi K, Tanaka S, Iida H, Eto H, Kato H, Aoi N, et al. Stromal vascular fraction isolated from lipo-aspirates using an automated processing system: bench and bed analysis. J Tissue Eng Regen Med 2013; 7: 864-70.
17 Domergue S, Bony C, Maumus M et al. Comparison between stromal vascular fraction and adipose mesenchymal stem cells in remodeling hypertrophic scars. PLoS One 2016; 11: e0156161

18 Han S, Sun HM, Hwang KC, Kim SW. Adipose-derived stromal vascular fraction cells: update on clinical utility and efficacy. Crit Rev Eukaryot Gene Expr 2015; 25: 145-52

19 Gupta MK, Ajay AK. Fat on sale: role of adipose-derived stem cells as antifibrosis agent in regenerative medicine. Stem Cell Res Ther 2015; 6: 233

20 You D, Jang MJ, Kim BH et al. Comparative study of autologous stromal vascular fraction and adipose-derived stem cells for erectile function recovery in a rat model of cavernous nerve injury. Stem Cells Transl Med 2015; 4: 351-8

21 Bowles AC, Strong AL, Wise RM et al. Adipose stromal vascular fraction-mediated improvements at late-stage disease in a murine model of multiple sclerosis. Stem Cells 2017; 35: 532-44

22 Castiglione F, Bergamini A, Russo A et al. Inhibition of phosphodiesterase 4 enhances clitoral and vaginal blood flow responses to dorsal clitoral nerve stimulation or PGE1 in anesthetized female rats. $J$ Sex Med 2013; 10: 939-50

23 Albersen M, Kendirci M, Van der Aa F, Hellstrom WJ, Lue TF, Spees JL. Multipotent stromal cell therapy for cavernous nerve injury-induced erectile dysfunction. J Sex Med. 2012; 9: 385-403

24 Castiglione F, Bergamini A, Albersen M et al. Pelvic nerve injury negatively impacts female genital blood flow and induces vaginal fibrosisimplications for human nerve-sparing radical hysterectomy. BJOG 2015; 122: $1457-65$

25 Castiglione F, Dewulf K, Hakim L et al. Adipose-derived stem cells counteract urethral stricture formation in rats. Eur Urol 2016; 70: 1032-41

26 Horton JA, Hudak KE, Chung EJ et al. Mesenchymal stem cells inhibit cutaneous radiation-induced fibrosis by suppressing chronic inflammation. Stem Cells 2013; 31: 2231-41

27 Zhu M, Dong Z, Gao J et al. Adipocyte regeneration after free fat transplantation: promotion by stromal vascular fraction cells. Cell Transplant 2015; 24: 49-62

28 Oberbauer E, Steffenhagen C, Wurzer C, Gabriel C, Redl H, Wolbank S. Enzymatic and non-enzymatic isolation systems for adipose tissue-derived cells: current state of the art. Cell Regen 2015; 4: 7

Correspondence: Petter Hedlund MD PhD, Division of Drug Research, Department of Medical and Health Sciences, Linküping University, 58183 Linküping, Svezia.

e-mail: petter.hedlund@med.lu.se

Abbreviations: ADSC, adipose tissue-derived stem cell; TA, tunica albuginea; PD, Peyronie's disease; SVF, stromal vascular fraction; ICP, intracavernosal pressure; MAP, mean arterial pressure; MSC, mesenchymal stem cell; ADSC, adipose tissue-derived mesenchymal stem cells; $\mathrm{CN}$, cavernous nerve; GADPH, glyceraldehyde 3-phosphate dehydrogenase. 